\title{
BCG VACCINE EFFECTIVENESS AGAINST BURULI ULCER: A CASE-CONTROL STUDY IN BENIN
}

\author{
FABIENNE NACKERS,* MICHÈLE DRAMAIX, ROCH CHRISTIAN JOHNSON, CLAUDE ZINSOU, ANNIE ROBERT, \\ ELISA DE BIURRUN BAKEDANO, JUDITH R. GLYNN, FRANÇOISE PORTAELS, AND RENÉ TONGLET $\dagger$ \\ Unité d'Epidemiologie, Ecole de Santé Publique, Université Catholique de Louvain, Brussels, Belgium; Laboratoire de Statistiques \\ Médicales, Université Libre de Bruxelles, Brussels, Belgium; Programme National de Lutte contre l'Ulcère de Buruli, Cotonou, Benin; \\ Centre de Dépistage et de Traitement de l'Ulcère de Buruli, Lalo, Benin; Centre Sanitaire et Nutritionnel Gbemoten, Zagnanado, \\ Benin; Infectious Disease Epidemiology Unit, London School of Hygiene and Tropical Medicine, London, United Kingdom; \\ Department of Microbiology, Institute of Tropical Medicine, Antwerp, Belgium
}

\begin{abstract}
BCG remains the only possible prophylactic intervention against Buruli ulcer (BU). Estimating its public health impact on BU control is an important issue. We conducted a case-control study to investigate the vaccine effectiveness of routine BCG vaccine against BU in southern Benin. From August 2002 to August 2003, BCG vaccination status was obtained for 279 clinically diagnosed BU cases and 988 age- and sex-matched neighborhood controls. BCG coverage, which was estimated by the presence of a scar or a vaccination record, was $64.5 \%$ in cases and $67.2 \%$ in controls. There was no evidence of a protective effect of routine BCG vaccination against BU in southern Benin (vaccine effectiveness adjusted for socioeconomic status $=12 \%, 95 \%$ confidence interval $=-24 \%$ to $37 \%$ ).
\end{abstract}

\section{INTRODUCTION}

In 1998, Mycobacterium ulcerans disease was declared by the World Health Organization as an emerging skin disease of public health concern. ${ }^{1}$ Usually called Buruli ulcer (BU), the disease has been reported in at least 30 (mostly tropical) countries. $^{2}$ The major burden is in west Africa ${ }^{3-5}$ where the clinical lesion starts as a painless subcutaneous nodule, plaque, or edema that secondarily ulcerates with characteristic undermined edges. ${ }^{6}$ Bones can also be involved. ${ }^{7}$ Although there is an increasing evidence of the efficacy of antibiotic therapy, treatment still relies on surgical excision of infected and necrotic tissues. Since BU mostly affects rural populations with limited geographic, financial, and cultural access to health services, patients usually present late with severe clinical forms of the disease, including extensive skin destruction, multiple lesions, secondary infection and/or bone involvement. Even with adequate treatment, permanent disabilities can remain.

Control strategies against BU are limited because of a lack of knowledge of potential risk factors and the absence of a specific vaccine. The protective effect of $\mathrm{BCG}$ vaccination in preventing BU remains controversial. Two trials conducted in Uganda ${ }^{8,9}$ reported short-lasting protection (47\%), and a case series in Benin suggested that BCG could protect against BU osteomyelitis. ${ }^{10,11}$ Conversely, case-control studies conducted in Ghana ${ }^{12,13}$ did not provide any evidence of a protective effect of BCG against BU. In a recent large hospital-based case-control study conducted in Benin, Debacker and oth$\mathrm{ers}^{14}$ reported an increased risk of BU after BCG vaccination in adults and children more than five years of age. Since BCG is the only prophylactic intervention, estimating its public health impact on BU control is an important issue.

The frequency of BU varies widely from village to village, even within the same district, ${ }^{15,16}$ which presumably reflects

* Address correspondence to Fabienne Nackers, Ecole de Santé Publique, Université Catholique de Louvain, Clos Chapelle-aux-Champs 30/EPID3058, 1200 Woluwé-Saint-Lambert, Belgium. E-mail: Fabienne.Nackers@epid.ucl.ac.be

$\dagger$ Deceased. differences in environmental exposure to M. ulcerans. Studies of risk factors for disease must take into account this variation in exposure. We present a case-control study with neighborhood controls to assess the effectiveness of routine BCG vaccination in preventing BU in southern Benin.

\section{METHODS}

Setting. The study was conducted in southern Benin. Buruli ulcer is endemic in this area with more than 5,700 patients reported since $1989 .^{2}$ In 2002, the area included two reference centers for the treatment of BU: the Center Sanitaire et Nutritionnel Gbemoten (CSNG) at Zagnanado, Zou region, and the Center de Dépistage et de Traitement de l'Ulcère de Buruli (CDT/UB) at Lalo, Mono-Couffo region. Patients come directly to these centers, often motivated by previously treated patients, and are also referred through village outreach activities. ${ }^{4}$ In 2003 , the prevalence of human immunodeficiency virus among Beninese adults 15-49 years of age was estimated to be between $1.1 \%$ and $3.3 \% .{ }^{17}$

Since the implementation of the Beninese Expanded Program of Immunization (EPI) in 1982, the vaccination policy is to administer BCG at birth. BCG had always been administered intradermally on the upper third of the dorsal surface of the left forearm. It was not possible to obtain information on the strains and doses used in Benin but it is likely that they varied over time.

Cases. From August 2002 to May 2003 at the CSNG and from August 2002 to August 2003 at the CDT/UB, all patients clinically diagnosed with BU by medical officers specialized in BU treatment were eligible for enrollment. Patients living outside Benin were excluded, as were those living in the cities of Cotonou and Porto-Novo, because it is unlikely that they contracted BU in those urban areas.

Controls. The study protocol aimed to recruit four controls per case. Neighborhood controls were individually matched to cases by sex and age (categories: < 1, 1-5, 6-12, 13-19, 20-29, 30-39, and $\geq 40$ years). A door-to-door systematic procedure was used for control selection. Field investigators started at the case's house, chose a random direction, and visited the nearest house. They listed all members of the 
household and identified potential controls fitting the matching criteria. If several subjects were identified, they chose the closest in age to the case. The procedure was repeated in each consecutive house until four appropriate controls were found. If identified potential controls were absent, one or two additional visits were planned. Controls were examined to rule out active or healed BU.

Data collection. Clinical information was abstracted from medical records. Lesions were classified according to clinical form: nodule, plaque, edema, ulcer, osteomyelitis, mixed, and other lesions. Specimens of tissue and of exudates from some patients were analyzed by Ziehl-Neelsen staining, culture, IS2404 polymerase chain reaction, and histopathologic examination $^{18}$ to confirm the clinical diagnosis. Participants were examined for a BCG scar. When available, vaccination records were checked. All subjects were asked about prior BCG vaccination. For children less than 15 years of age, parents or guardians were used as informants. Evidence of BCG vaccination was defined by a BCG scar or a BCG vaccination record. Individuals with a doubtful scar were excluded from the analysis. In a separate analysis, we also excluded subjects without a scar or vaccination record but who provided a history of BCG vaccination. Scar size was not recorded.

Participants or guardians answered a questionnaire. Questions on socioeconomic characteristics included education level of the participants, education level and occupation of the head of the participant's household, type of house, and possession of selected items by the household (radio, bicycle, motorbike, car, and dugout canoe). The household was defined as all subjects sharing the same roof and the same cooking pot. Factors recorded to estimate likely environmental exposure to $M$. ulcerans included participation in farming, water-contact frequency with distinction between stagnant and flowing water, and main drinking water source and drawing water at this source. Information on whether young children accompanied adults during farming or obtaining water was recorded.

Interviews were conducted in French, Fon, or Adja by nurses or biomedical technicians trained in the study protocol, BCG scar reading and recognizing BU lesions. Hospital and field teams included different investigators not blind to the disease status of the participants.

Statistical analysis. Estimating from a preliminary pilot study that $25 \%$ of the controls and $35 \%$ of the cases had no BCG scar, the proportion of discordant pairs was expected to be $43 \%$. To have $80 \%$ power to detect whether the odds ratio (OR) is significantly different from 1 at the $5 \%$ level, with a VE of $40 \%$ considered to be clinically relevant, the sample should include 208 cases and 832 matched-controls in a 1:4 matched ratio. ${ }^{19}$ In practice, we aimed to recruit 300 cases to allow subgroup analysis.

The ORs and $95 \%$ confidence intervals (CIs) were calculated using conditional logistic regression. Associations were tested using the likelihood ratio test (LRT) and, for ordered categorical variables, the LRT for trend. Vaccine effectiveness $(\mathrm{VE} \%)$ was calculated as $(1-\mathrm{OR}) \times 100 .{ }^{20}$ Estimation of VE was adjusted for socioeconomic status (SES) and for factors estimating likely environmental exposure to $M$. ulcerans; age, sex, and neighborhood were accounted for by the matched design and analysis. The SES was measured by house type, education level of the head of the household, and a score of ownership based on household possession of a radio, bicycle, motorbike, dugout canoe, or car. Items were weighted according to the inverse frequency of households owning this item in rural Benin in $2001^{21}$ and then summed. Higher scores therefore reflected both greater ownership and owning less common (and by inference more valuable) items. Vaccine effectiveness was assessed separately for age groups older and younger than 20 years (born after the implementation of the EPI and therefore likely to have been vaccinated at birth), by sex, for laboratory confirmed BU, and for patients with BU osteomyelitis. Differences between subgroups were tested for interaction using LRT. Reliability of scar reading was estimated using a kappa coefficient for multiple observers. Data were entered in Epi-Info version 6.04 (Centers for Disease Control and Prevention, Atlanta, GA) and analyzed with STATA version 9.0 (Stata Corporation, College Station, TX).

Ethics. Data were collected after informed consent of the participant or participant's guardian. Potential controls with BU lesions were offered appropriate treatment. The study was reviewed and approved by the Beninese Public Health Minister.

\section{RESULTS}

Study population. Among the 446 eligible patients, 2 refused participation in the study, 3 were excluded because of doubtful scars, 1 was excluded because of missing BCG data, 42 were excluded because a BU lesion on the left forearm made identification of a BCG scar impossible, and $78(17.5 \%)$ were not recruited because of work overload in the health centers. Information on BCG status was thus available for 320 patients. Mainly because of wrongly listed addresses and impassable roads preventing field teams from reaching villages, suitable controls could be matched for only $229(71.6 \%)$ of these patients. Fifty patients hospitalized for BU at the CSNG between January and August 2002 and living in villages vis-

TABLE 1

Description of the cases $(n=279)$ of Buruli ulcer in Benin

\begin{tabular}{lrr}
\hline \multicolumn{1}{c}{ Variable } & No. & $\%$ \\
\hline Clinical form $\dagger$ & & \\
Edema & 8 & 2.9 \\
Ulcer & 127 & 45.7 \\
Nodule & 7 & 2.5 \\
Plaque & 61 & 21.9 \\
Osteomyelitis & 7 & 2.5 \\
Others & 3 & 1.1 \\
Mixed & 65 & 23.4 \\
Clinical stage $\dagger$ & & \\
Not ulcerative & 92 & 33.1 \\
Ulcerative & 137 & 49.3 \\
Healing & 34 & 12.2 \\
Several stages & 12 & 4.3 \\
Others & 3 & 1.1 \\
Number of positive laboratory test results $\neq$ & & \\
0 & 45 & 16.1 \\
1 & 34 & 12.2 \\
$\geq 2$ & 100 & 35.8 \\
Untested & 100 & 35.8 \\
Hospitalized at the CDT/UB, Lalo* & 92 & 33.0 \\
Hospitalized at the CSNG, Zagnanado* & 187 & 67.0 \\
\hline * CDT/UB = Centre de Dépistage et de Traitement de l’Ulcère de Buruli; CSNG & & \\
Centre Sanitaire et Nutritionnel Gbemoten. & & \\
$\dagger$ One missing value. & \\
\# Ziehl-Neelsen staining, histology, culture and/or polymerase chain reaction. & \\
\end{tabular}


TABLE 2

Demographic characteristics of the participants

\begin{tabular}{lccc}
\hline & $\begin{array}{c}\text { Cases } \\
\text { No. }(\%) \\
\mathrm{n}=279\end{array}$ & $\begin{array}{c}\text { Controls } \\
\text { No. }(\%) \\
\mathrm{n}=988\end{array}$ & $P$ \\
\hline Female & $131(47.0)$ & $467(47.3)$ & $*$ \\
Age (years) & $36(12.9)$ & $114(11.5)$ & $*$ \\
$<6$ & $99(35.5)$ & $360(36.4)$ & \\
6 to $<13$ & $36(12.9)$ & $136(13.8)$ & \\
13 to $<20$ & $39(14.0)$ & $133(13.5)$ & \\
20 to $<30$ & $18(6.5)$ & $72(7.3)$ & \\
30 to $<40$ & $51(18.3)$ & $173(17.5)$ & $*$ \\
$\geq 40$ & $14(5.0)$ & $43(4.4)$ & \\
Region of residence & $122(43.7)$ & $429(43.4)$ & \\
$\quad$ Atlantique & $52(18.6)$ & $179(18.1)$ & \\
Ouémé-Plateau & $91(32.6)$ & $337(34.1)$ & \\
Zou & & & \\
$\quad$ Mono-Couffo & $222(80.1)$ & $792(80.7)$ & \\
Ethnicity $\dagger$ & $48(17.3)$ & $169(17.2)$ & \\
$\quad$ Fon & $7(2.5)$ & $21(2.1)$ & \\
Adja & & & \\
Others & &
\end{tabular}

* Matching variable.

$\dagger$ Two matched sets lost due to missing data in cases.

\$ By conditional logistic regression.

ited by the field investigators were retrospectively recruited with suitable controls. They were included in the analysis, resulting in a total of 279 matched sets. Cases included in the matched analysis were similar to the eligible cases not included regarding sex and age, but they were more likely to have come from the Ouémé region and to be vaccinated with BCG $(64.5 \%$ versus $50.5 \% ; P=0.018)$.

In 7 of more than 200 villages visited, local authorities refused to allow persons to participate in the study. Eight controls were reported to have refused to answer the question- naire but individual refusals were not systematically recorded. Three controls with suspected BU lesions, 3 with missing BCG data, and 25 with doubtful scars were excluded. A total of 988 controls were matched to the 279 cases (average matching ratio $=1: 3.5$ ).

Clinical description of cases. The median age was 13.4 years (range $=11$ months to 80 years) and $47 \%$ were female. Ulcer was the most frequent form $(46 \%)$ followed by the mixed $(23 \%)$ and the plaque forms $(22 \%)$ (Table 1). A total of 24 $(8.7 \%)$ patients had bone involvement ( 7 without active skin lesions and 17 with a mixed form of BU). Recurrence accounted for $21(7.5 \%)$ of the cases. Forty-eight percent of the cases were confirmed by at least one of the four laboratory tests (Table 1).

Sociodemographic characteristics and SES. Matching resulted in sex, age, and region of residence being similar in cases and controls (Table 2). The predominant ethnic group was Fon. Cases were more likely than controls to live in cemented or brick houses and were of higher SES as measured by the score of ownership and education level of the head of the household (Table 3). However, there was no evidence that education level of cases and controls more than 20 years of age differed (Table 3). Among those $6-19$ years of age, $83(61.9 \%)$ of the cases were currently attending school compared with $306(66.0 \%)$ of the controls $(P=0.38)$.

BCG vaccination coverage. $B C G$ coverage estimated by the presence of a scar or a vaccination record was $64.5 \%$ among 279 cases and $67.2 \%$ among 988 controls. As shown in Table 4, 11 participants with a scar or a BCG vaccination record claimed not to have been vaccinated. Thirty $(30.3 \%)$ of 99 cases and 117 (36.1\%) of 323 controls without a scar or vaccination record claimed to have been vaccinated. Overall,

TABLE 3

Socioeconomic characteristics of the participants

\begin{tabular}{|c|c|c|c|c|}
\hline & $\begin{array}{c}\text { Cases } \\
\text { No. }(\%)\end{array}$ & $\begin{array}{l}\text { Controls } \\
\text { No. }(\%)\end{array}$ & OR $(95 \% \mathrm{CI})^{*}$ & $P_{\dagger}^{\dagger}$ \\
\hline House type & & & & $<0.001$ \\
\hline Mud, wood, straw & 75 (27.3) & $369(38.5)$ & 1 & \\
\hline Mud, sheet metal roof & $139(50.6)$ & $538(56.2)$ & $1.49(1.03,2.14)$ & \\
\hline Cement, brick & $61(22.2)$ & $51(5.3)$ & $10.04(5.64,17.84)$ & \\
\hline Head of the household's education level & & & & $0.006+$ \\
\hline None & $149(56.9)$ & $581(64.3)$ & 1 & \\
\hline Primary uncompleted & $59(22.5)$ & $190(21.0)$ & $1.30(0.81,1.83)$ & \\
\hline Completed primary or higher & $54(20.6)$ & $132(14.6)$ & $1.77(1.19,2.59)$ & \\
\hline Participant's education level (> 20 years) & & & & 0.43 \\
\hline None & $72(67.9)$ & $273(73.8)$ & 1 & \\
\hline Primary uncompleted & $20(18.9)$ & $55(14.9)$ & $1.51(0.79,2.91)$ & \\
\hline Completed primary or higher & $14(13.2)$ & $42(11.4)$ & $1.44(0.62,3.34)$ & \\
\hline Head of the household's occupation & & & & 0.028 \\
\hline Farmer, fisher & $167(61.9)$ & $661(70.8)$ & 1 & \\
\hline Manual, trade, driver & $63(23.3)$ & $189(20.2)$ & $1.35(0.93,1.96)$ & \\
\hline Professional & $24(8.9)$ & $53(5.7)$ & $2.02(1.13,3.64)$ & \\
\hline Village/religious authority, traditional healer & $6(2.2)$ & $17(1.8)$ & $1.53(0.56,4.13)$ & \\
\hline Retired, none & $10(3.7)$ & $14(1.5)$ & $3.15(1.16,8.54)$ & \\
\hline Score of ownership§ & & & & $<0.001 \ddagger$ \\
\hline 1 & $28(10.4)$ & $159(17.1)$ & 1 & \\
\hline 2 & $91(33.7)$ & $400(42.9)$ & $1.40(0.85,2.30)$ & \\
\hline 3 & $101(37.4)$ & $287(30.8)$ & $2.20(1.33,3.64)$ & \\
\hline 4 & $50(18.5)$ & $86(9.2)$ & $4.36(2.40,7.95)$ & \\
\hline
\end{tabular}


TABLE 4

$\mathrm{BCG}$ vaccination status of cases and controls according to the source of information

\begin{tabular}{lrrrr}
\hline & \multicolumn{3}{c}{$\begin{array}{c}\text { History of BCG vaccination } \\
\text { according to the subject } \\
\text { or subject's guardian* }\end{array}$} \\
\cline { 2 - 5 } $\begin{array}{c}\text { Clinical examination for a BCG scan } \\
\text { and BCG vaccination record }\end{array}$ & Yes & $\begin{array}{c}\text { Do not } \\
\text { know }\end{array}$ & Total \\
\hline Cases & 148 & 4 & 12 & 164 \\
Presence of a BCG scar & 15 & 1 & 0 & 16 \\
BCG vaccination record ( \pm BCG scar) & 30 & 59 & 10 & 99 \\
Not BCG vaccinated (no scar, no record) & 193 & 64 & 22 & 279 \\
Total & & & & \\
Controls & 567 & 6 & 44 & 617 \\
Presence of a BCG scar & 43 & 0 & 3 & 46 \\
BCG vaccination record ( \pm BCG scar) \\
Not BCG vaccinated (no scar, no record) \\
Total & 117 & 158 & 48 & 323 \\
\hline * Missing value in one control with a BCG scar and one control without BCG scar or BCG \\
vaccination record.
\end{tabular}

$84.0 \%$ of the 920 participants claiming to have been vaccinated with BCG had a recognizable scar or a BCG vaccination record.

Sixty-two subjects (16 cases and 46 controls) had a BCG vaccination record. They were less than 12 years of age and were vaccinated before five months of age. Scar examination in two of them was not possible because the BU lesion was on the vaccination site; $13.3 \%$ ( 8 of 60 ) had no scar.

A subsample of subjects were examined independently for BCG scar by four investigators of CSNG $(n=53)$ and three investigators of CDT/UB $(\mathrm{n}=27)$. Kappa coefficients were 0.78 for CSNG and 0.90 for $\mathrm{CDT} / \mathrm{UB}$, which indicated good intra-observer agreement on the presence of a scar.

BCG vaccine effectiveness. The BCG VE was $15 \%(95 \%$ $\mathrm{CI}=-15 \%$ to $37 \%, P=0.29$ ) (Table 5 ). When adjusted for SES, as measured by education level of the head of the household, score of ownership and type of house, VE changed very little (Table 5). Adjusting for head of the household's occupation or for the participant's education level did not change the estimates. The estimates were also unchanged after additionally adjusting for factors measuring likely environmental exposure to $M$. ulcerans (contacts with flowing water, contacts with stagnant water, participation in farming, and drawing surface water for drinking).

Few participants had more than one BCG scar and the trend for an increased VE with increasing number of doses was not statistically significant (Table 5). Excluding subjects without scar or vaccination record but reporting a history of BCG vaccination increased the VE estimations (Table 5) but still provided weak evidence for a protective effect of BCG against BU.

Table 6 shows VE estimations for patient subgroups. The VE was higher in subjects more than 20 years of age but CIs were wide, and the test for interaction did not provide evidence for a true difference compared with $\mathrm{VE}$ in younger people. Also, the VE was higher in females than in males, but the interaction was not statistically significant. The VE was similar in laboratory-confirmed cases and unconfirmed cases. The VE tended to be higher against BU osteomyelitis compared with other forms of $\mathrm{BU}$, but there was no statistical evidence for a difference, as reflected by the overlapping $95 \%$ CIs.

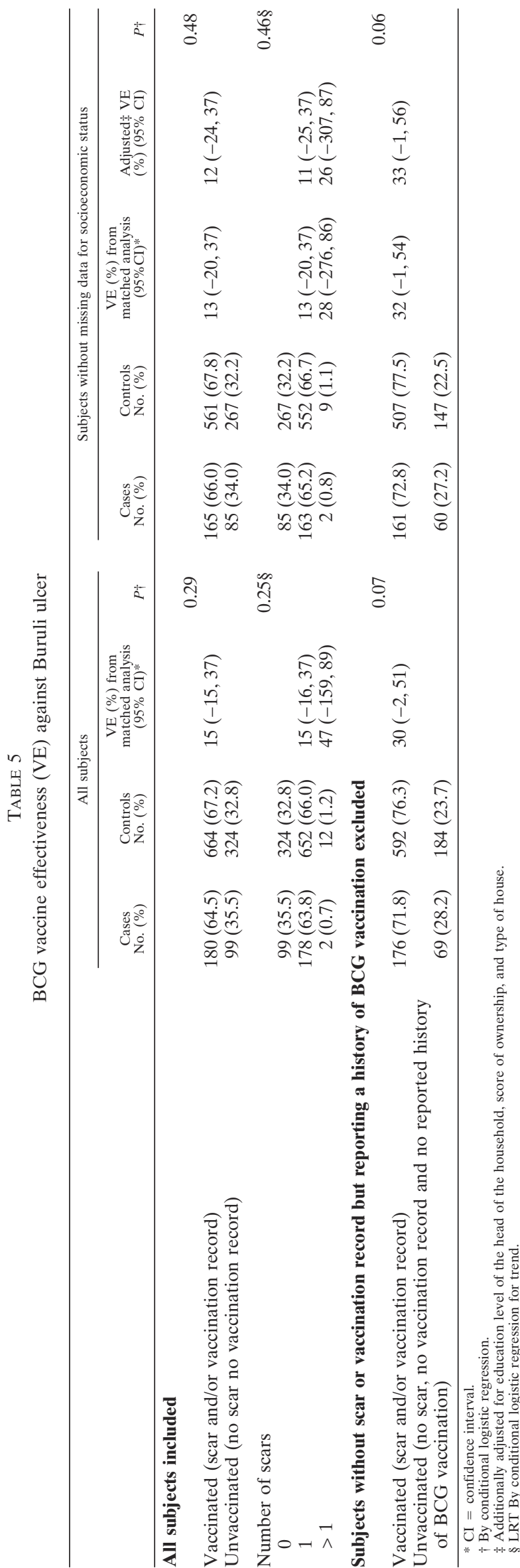


TABLE 6

BCG vaccine effectiveness (VE) against Buruli ulcer (BU) by age group and sex for confirmed BU and BU osteomyelitis

\begin{tabular}{|c|c|c|c|c|}
\hline & $\begin{array}{l}\text { Cases } \\
\text { No. }(\% \text { vaccinated }) \dagger\end{array}$ & $\begin{array}{l}\text { Controls } \\
\text { No. }(\% \text { vaccinated }) \dagger\end{array}$ & $\begin{array}{l}\mathrm{VE}(\%) \text { from matched } \\
\text { analysis }(95 \% \mathrm{CI})^{*}\end{array}$ & $\begin{array}{l}\text { Adjusted } \$ \mathrm{VE}(\%) \\
(95 \% \mathrm{CI})^{*}\end{array}$ \\
\hline \multicolumn{5}{|l|}{ Age, years ${ }^{\mathrm{a}}$} \\
\hline$<20$ & $171(71.4)$ & $610(72.1)$ & $7(-37,38)$ & $-7(-67,32)$ \\
\hline$\geq 20$ & $108(53.7)$ & $378(59.3)$ & $24(-21,53)$ & $33(-15,60)$ \\
\hline \multicolumn{5}{|l|}{$\operatorname{Sex}^{\mathrm{b}}$} \\
\hline Female & $131(58.8)$ & 467 (66.4) & $33(-3,57)$ & $29(-18,57)$ \\
\hline Male & $148(69.6)$ & $521(68.0)$ & $-6(-62,30)$ & $-6(-67,34)$ \\
\hline Confirmed BU§ & $134(67.9)$ & 465 (71.6) & $21(-25,50)$ & $14(-45,49)$ \\
\hline Not confirmed BU & 145 (61.4) & $523(63.3)$ & $10(-33,40)$ & $10(-42,42)$ \\
\hline BU osteomyelitis & $24(50.0)$ & $78(64.1)$ & $48(-48,81)$ & $53(-34,83)$ \\
\hline Other BU & $253(66.0)$ & $902(67.7)$ & $12(-21,36)$ & $6(-36,35)$ \\
\hline
\end{tabular}

* $\mathrm{CI}=$ confidence interval.

a $\mathrm{P}$ values (conditional logistic regression) for modification of $\mathrm{VE}$ according to age groups $=0.52$ (matched analysis) and 0.19 (adjusted for socioeconomic status [SES]).

${ }^{\mathrm{b}} \mathrm{P}$ values (conditional logistic regression) for modification of VE according to sex $=0.13$ (matched analysis) and 0.26 (adjusted for SES).

$\dagger$ Vaccinated $=$ scar and/or vaccination record; unvaccinated $=$ no scar and no vaccination record.

\$ Adjusted for SES: head of the household's education level, score of ownership, and type of house.

$\S$ Confirmed by at least one laboratory test (Ziehl-Neelsen staining, culture and IS2404 polymerase chain reaction and histopathologic examination).

\section{DISCUSSION}

Our study does not provide evidence that BCG administered under routine conditions in Benin reduces the risk of BU among those vaccinated. Overall VE estimation adjusted for SES was $12 \%$ ( $95 \% \mathrm{CI}=-24 \%$ to $37 \%)$. Although the CI is wide, strong protection is unlikely.

Our results are consistent with those of two case-control studies conducted in Ghana. ${ }^{12,13}$ Those studies were designed to investigate several potential risk factors for BU, including presence of a BCG scar. Although the investigators did not report the OR (or VE), they reported no statistically significant difference in BCG scar prevalence between cases and matched neighborhood controls of similar age, as in our study.

In two Ugandan trials, ${ }^{8,9} \mathrm{VE}$ against $\mathrm{BU}$ was estimated to be $72 \%$ in the first 6 months $^{8}$ and $63 \%$ in the first year ${ }^{9}$ after vaccination; it then waned to $0 \%$. BCG was administered to subjects (from less than one to more than 25 years of age) with weak or negative tuberculin reactions. Besides important differences in geographic location and design, our results are difficult to compare with those trials because most of our participants received neonatal BCG and were included in the study more than one year after vaccination. Also, those studies estimated vaccine efficacy under ideal storage, handling, and administration conditions of a clinical trial, while our study estimated vaccine effectiveness, i.e., efficacy under field conditions. $^{22}$

Because of the small area variation in the distribution of $M$. ulcerans $^{15,16}$ and the likely geographic variation of BCG vaccine coverage, we used neighborhood controls. We were able to adjust for factors linked to likely environmental exposure to M. ulcerans, and this did not change our estimates. Neighborhood controls have the disadvantage of possibly being less frequent users of health services. At the time of our study, the CSNG and CDT/UB were the main referral centers for treating BU in southern Benin. Both centers had important village outreach activities and some patients were referred to the centers through active case detection. Consequently, referral patterns of BU patients differed from those of patients treated for other diseases in the same centers. However, ascertainment of BU cases in the region is unlikely to have been complete because people are often reluctant to seek medical treatment of $\mathrm{BU},{ }^{23}$ mainly because of fear of surgery and the cost of treatment, especially the indirect costs during the long duration of hospitalization. ${ }^{24}$ We adjusted VE for SES to minimize this bias in likely health service usage patterns between cases and controls and VE changed very little. However, SES is difficult to measure and we cannot exclude residual confounding.

Because of work overload in the health centers, not all eligible patients were recruited, but this was regardless of their BCG status. For those recruited, matching was incomplete mainly because of wrongly listed addresses and impassable roads that prevented field teams from reaching villages. Included cases had a similar age and sex distribution compared with those not included, but included cases came from different geographic areas than case not included. In particular, included cases were more likely to come from the OuéméPlateau region where the highest BCG coverage has been reported. ${ }^{21}$ Also, BCG coverage is likely to be lower in difficult to reach areas. Consequently, $64.5 \%$ of the cases included in the matched analysis were vaccinated compared with $50.5 \%$ of those not included. Since controls were matched in neighborhoods, this would not have biased our VE estimation.

Only half of the cases were laboratory confirmed and misclassified cases can decrease VE estimation. ${ }^{25}$ However, there was no statistical evidence for a difference in BCG VE between laboratory confirmed cases and other cases.

Uncertainty about BCG vaccination status could have led to underestimation of BCG $\mathrm{VE}$ in our study. Vaccination records were seldom available and our definition of BCG vaccination status relied mainly on the presence of a BCG scar. Presence of a scar is recognized as a highly sensitive and reliable indicator of $\mathrm{BCG}$ vaccination status ${ }^{26}$ except when administered within one month of birth. ${ }^{27}$ Neonatal vaccination is a long-established policy in Benin, and we could estimate in our study that approximately $87 \%$ of the BCG vaccinations resulted in a scar. Since scar formation is probably independent of immune response, ${ }^{27}$ this could have led to non-differential misclassification and VE underestimation. Therefore, we excluded subjects without BCG scars or vaccination records but who claimed to have received $\mathrm{BCG}$ and re-estimated BCG VE. Vaccine effectiveness estimates in- 
creased to $33 \%$ (SES-adjusted) but the CI was wide and still provided weak statistical evidence for an effect of BCG in preventing BU in Benin.

Cases and controls were examined by different investigators. They were not blinded to the disease status of the participant or to the research hypothesis. Therefore, we cannot formally exclude differential observer bias. However, in both centers, teams were trained by the same member of the research team, and we have no reasons to believe that cases were more carefully examined for scars than controls.

The benefit of BCG revaccination had been reported against leprosy, in a setting where the initial dose appeared protective, $^{28}$ but not against tuberculosis. ${ }^{28,29}$ In Uganda, there was no evidence for an enhanced protection of BCG revaccination against $\mathrm{BU} .{ }^{9}$ We observed a trend for an increased VE in subjects with more than one scar attributable to BCG vaccination. However, few subjects had more than one scar and the trend was not statistically significant. We also found a higher protective effect of BCG against BU osteomyelitis, which is consistent with the observations of Portaels and others, ${ }^{10,11}$ but the numbers of patients were small.

We found higher VE for subjects more than 20 years of age compared with younger persons. This is surprising because $\mathrm{VE}$ is expected to wane with time since vaccination, ${ }^{30}$ and VE estimation in subjects more than 20 years of age measured the long-lasting protection conferred by BCG administered at least 20 years previously. Underestimation of $\mathrm{VE}$ could be serious if less than $90 \%$ of the vaccinations left a scar and more than $70 \%$ of the population has been vaccinated, ${ }^{31}$ which is the case for subjects younger than 20 years of age included in our study. This underestimation could therefore have been more important in this group than in the older age group, in whom vaccination coverage was less than $60 \%$ and vaccines may have been given beyond the neonatal period. However, there was no statistical evidence for a true difference of BCG VE between age groups.

Although our BCG VE estimations could have been slightly underestimated, our data do not provide evidence of a strong protective effect of routinely administered BCG against BU in southern Benin. From a public health perspective, routine neonatal BCG appears of little value in controlling BU in this population. Thus, effective prevention of BU by immunoprophylaxis needs further vaccine development.

Received March 31, 2006. Accepted for publication June 1, 2006.

Acknowledgments: This article is dedicated to the memory of Professor René Tonglet, who passed away June 5, 2005. We sincerely thank the field investigators, the staff of the Programme National de Lutte contre de l'Ulcère de Buruli, CDT/UB, and CSNG for their help and hospitality, especially Soeur Julia Aguiar. We are grateful to all patients and villagers for participating in this study.

Financial support: This work was supported by the Damien Foundation (Brussels, Belgium), the Belgian Fonds de la Recherche Scientifique Médicale (grant no. 3.4501.03), and the Université Catholique de Louvain Fonds Spéciaux de Recherche (grant No 2002, 20c5). Fabienne Nackers analyzed the data while obtaining an MSc degree in Epidemiology at the London School of Hygiene and Tropical Medicine. She was supported by a British Medical Research Council Advanced Course Masters studentship and a grant from the Belgian Medical Foundation Mathilde Horlait-Dapsens. Roch Christian Johnson, Claude Zinsou, and Elisa de Biurrun Bakedano were supported by a grant from the Directorate-General for Development Cooperation (Brussels, Belgium; Project: Buruli Ulcer in Benin). Judith Glynn is supported by the United Kingdom Department of Health (Public Health Career Scientist Award).
Authors' addresses: Fabienne Nackers, Annie Robert, and René Tonglet, Ecole de Santé Publique, Université Catholique de Louvain, Clos Chapelle-aux-Champs 30/EPID3058, 1200 Woluwé-SaintLambert, Belgium, Telephone: 32-2-764-3320, Fax: 32-2-764-3328, E-mails: Fabienne.Nackers@epid.ucl.ac.be and Annie.Robert@epid .ucl.ac.be. Michèle Dramaix, Ecole de Santé Publique, Université Libre de Bruxelles, Route de Lennik 808, 1070 Brussels, Belgium, Telephone: 32-2-555-4051, Fax: 32-2-555-4049, E-mail: miwilmet@ ulb.ac.be. Roch Christian Johnson, Programme National de Lutte contre l'Ulcère de Buruli au Bénin, Ministère de la Santé Publique, 06 BP 2572, Cotonou, Benin, Telephone: 229-331-827, Fax: 229-337057, E-mail: rochjohnson@yahoo.fr. Claude Zinsou, Centre Sanitaire et Nutritionnel de Gbemoten, Zagnanado, Benin, Fax: 229-520-293. Elisa de Biurrun Bakedano, Centro Nacional de Medicina Tropical, Instituto de Salud Carlos III, C/Sinesio Delgado 4, Pabellón 13, 28029 Madrid, Spain, Telephone: 91-8-222-940, Fax: 91-3-877-756, E-mail: ebiurrun@isciii.es. Judith R. Glynn, Infectious Disease Epidemiology Unit, London School of Hygiene and Tropical Medicine, Keppel Street, London WC1E 7HT, United Kingdom, Telephone: 44-20-79272423, Fax: 44-20-7636-8739, E-mail: Judith.Glynn@lshtm.ac.uk. Françoise Portaels, Department of Microbiology, Institute of Tropical Medicine, Nationalestraat 155, 2000 Antwerp, Belgium, Telephone: 32-3-247-6317, Fax: 32-3-247-6333, E-mail: portaels@itg.be.

\section{REFERENCES}

1. World Health Organization. The Yamoussoukro Declaration on Buruli Ulcer. Accessed March 2006. Available from http:// www.who.int/buruli/yamoussoukro_declaration/en/index.html.

2. Buruli ulcer disease, 2004. Wkly Epidemiol Rec 79: 194-199.

3. Amofah G, Bonsu F, Tetteh C, Okrah J, Asamoa K, Asiedu K, Addy J, 2002. Buruli ulcer in Ghana: results of a national case search. Emerg Infect Dis 8: 167-170.

4. Debacker M, Aguiar J, Steunou C, Zinsou C, Meyers WM, Guedenon A, Scott JT, Dramaix M, Portaels F, 2004. Mycobacterium ulcerans disease (Buruli ulcer) in rural hospital, southern Benin, 1997-2001. Emerg Infect Dis 10: 1391-1398.

5. Marston BJ, Diallo MO, Horsburgh CR Jr, Diomande I, Saki MZ, Kanga JM, Patrice G, Lipman HB, Ostroff SM, Good RC, 1995. Emergence of Buruli ulcer disease in the Daloa region of Côte d'Ivoire. Am J Trop Med Hyg 52: 219-224.

6. van der Werf TS, van der Graaf WT, Tappero JW, Asiedu K, 1999. Mycobacterium ulcerans infection. Lancet 354: 10131018.

7. Portaels F, Zinsou C, Aguiar J, Debacker M, Biurrun Bakedano E, Guedenon A, Josse R, Lagarrigue V, Silva M, Steunou C, Meyers WM, 2003. Les atteintes osseuses dans l'ulcère de Buruli: à propos de 73 cas. Bull Seances Acad R Sci Outre Mer 49: 161-190.

8. BCG vaccination against Mycobacterium ulcerans infection (Buruli ulcer). First results of a trial in Uganda, 1969. Lancet 1: 111-115.

9. Smith PG, Revill WD, Lukwago E, Rykushin YP, 1977. The protective effect of BCG against Mycobacterium ulcerans disease: a controlled trial in an endemic area of Uganda. Trans $R$ Soc Trop Med Hyg 70: 449-457.

10. Portaels F, Aguiar J, Debacker M, Steunou C, Zinsou C, Guédénon A, Meyers WM, 2002. Prophylactic effect of Mycobacterium bovis BCG vaccination against osteomyelitis in children with Mycobacterium ulcerans disease (Buruli ulcer). Clin Diagn Lab Immunol 9: 1389-1391.

11. Portaels F, Aguiar J, Debacker M, Guedenon A, Steunou C, Zinsou C, Meyers WM, 2004. Mycobacterium bovis BCG vaccination as prophylaxis against Mycobacterium ulcerans osteomyelitis in Buruli ulcer disease. Infect Immun 72: 62-65.

12. Amofah GK, Sagoe-Moses C, Adjei-Acquah C, Frimpong EH, 1993. Epidemiology of Buruli ulcer in Amansie West district, Ghana. Trans R Soc Trop Med Hyg 87: 644-645.

13. Raghunathan PL, Whitney EA, Asamoa K, Stienstra Y, Taylor TH Jr, Amofah GK, Ofori-Adjei D, Dobos K, Guarner J, Martin S, Pathak S, Klutse E, Etuaful S, van der Graaf WT, van der Werf TS, King CH, Tappero JW, Ashford DA, 2005. 
Risk factors for Buruli ulcer disease (Mycobacterium ulcerans Infection): results from a case-control study in Ghana. Clin Infect Dis 40: 1445-1453.

14. Debacker M, Portaels F, Aguiar J, Steunou C, Zinsou C, Meyers WM, Dramaix M, 2006. Risk factors for Buruli ulcer, Benin. Emerg Infect Dis. Available at http://www.cdc.gov/ncidod/ EID/vol12no09/05-0598htm.

15. Johnson RC, Makoutode M, Sopoh GE, Elsen P, Gbovi J, Pouteau LH, Meyers WM, Boko M, Portaels F, 2005. Buruli ulcer distribution in Benin. Emerg Infect Dis 11: 500-501.

16. Stoffel V, Barthelme B, Chague F, 2005. Tropical ecopathology: up hill and down dale Buruli ulcer. Sante Publique 17: 191-197.

17. UNAIDS, 2004. UNAIDS/WHO Epidemiological Fact Sheet. Benin. 2004 Update. Geneva: UNAIDS/WHO.

18. World Health Organization, 2001. Buruli ulcer. Diagnosis of Mycobacterium ulcerans Disease. A Manual for Health Care Providers. Portaels F, Johnson P, Meyers WM, editors. Geneva: World Health Organization.

19. Machin D, Campbell M, Fayers P, Pinol A, 1997. Sample Size Tables for Clinical Studies. Second edition. Oxford, United Kingdom: Blackwell Science Ltd.

20. Smith PG, 1988. Epidemiological methods to evaluate vaccine efficacy. Br Med Bull 44: 679-690.

21. Institut National de la Statistique et l'Analyse Economique (INSAE) et ORC Macro, 2002. Enquête Démographique et de Santé au Bénin 2001. Calverton, MD: Institut National de la Statistique et l'Analyse Economique (INSAE) et ORC Macro.

22. Comstock GW, 1994. Evaluating vaccination effectiveness and vaccine efficacy by means of case-control studies. Epidemiol Rev 16: 77-89.

23. Aujoulat I, Johnson C, Zinsou C, Guedenon A, Portaels F, 2003. Psychosocial aspects of health seeking behaviours of patients with Buruli ulcer in southern Benin. Trop Med Int Health 8: 750-759.

24. Asiedu K, Etuaful S, 1998. Socioeconomic implications of Buruli ulcer in Ghana: a three-year review. Am J Trop Med Hyg 59: 1015-1022.

25. Rodrigues LC, Smith PG, 1999. Use of the case-control approach in vaccine evaluation: efficacy and adverse effects. Epidemiol Rev 21: 56-72.

26. Pereira SM, Dourado I, Barreto ML, Cunha SS, Ichiara MY, Hijjar MA, Goes JC, Rodrigues LC, 2001. Sensitivity and specificity of BCG scar reading in Brazil. Int J Tuberc Lung Dis 5: 1067-1070.

27. Floyd S, Ponnighaus JM, Bliss L, Warndorff DK, Kasunga A, Mogha P, Fine PE, 2000. BCG scars in northern Malawi: sensitivity and repeatability of scar reading, and factors affecting scar size. Int J Tuberc Lung Dis 4: 1133-1142.

28. Karonga Prevention Trial Group, 1996. Randomised controlled trial of single $\mathrm{BCG}$, repeated $\mathrm{BCG}$, or combined $\mathrm{BCG}$ and killed Mycobacterium leprae vaccine for prevention of leprosy and tuberculosis in Malawi. Lancet 348: 17-24.

29. Rodrigues LC, Pereira SM, Cunha SS, Genser B, Ichihara MY, de Brito SC, Hijjar MA, Dourado I, Cruz AA, Sant'Anna C, Bierrenbach AL, Barreto ML, 2005. Effect of BCG revaccination on incidence of tuberculosis in school-aged children in Brazil: the BCG-REVAC cluster-randomised trial. Lancet 366 : 1290-1295.

30. Sterne JA, Rodrigues LC, Guedes IN, 1998. Does the efficacy of BCG decline with time since vaccination? Int J Tuberc Lung Dis 2: 200-207.

31. Smith PG, 1982. Retrospective assessment of the effectiveness of BCG vaccination against tuberculosis using the case-control method. Tubercle 63: 23-35. 